\title{
Pediatric sedation using dexmedetomidine and remimazolam for magnetic resonance imaging
}

\author{
Naohiro Shioji ${ }^{1,2} \cdot$ Tobias Everett $^{3} \cdot$ Yasuyuki Suzuki $^{4} \cdot$ Kazuyoshi Aoyama $^{3,5}(0)$
}

Received: 25 May 2021 / Accepted: 2 June 2021 / Published online: 5 June 2021

C Japanese Society of Anesthesiologists 2021

Keywords Magnetic resonance imaging $\cdot$ Pediatric anesthesia $\cdot$ Sedation $\cdot$ Dexmedetomidine $\cdot$ Remimazolam

The use of magnetic resonance imaging (MRI) in the pediatric population is expanding. Children usually need general anesthesia or sedation to be still for adequate MRI images. While dexmedetomidine has potential benefit for pediatric MRI sedation because of preservation of airway tone, there is not currently consensus for its use for pediatric MRI sedation. In this issue of Journal of Anesthesia, Kim et al. conducted a systematic review and meta-analysis of randomized controlled trials [1], in which they assessed the efficacy and safety of dexmedetomidine sedation for pediatric MRI compared to propofol, ketamine, and midazolam. The authors showed that compared to propofol, ketamine, and midazolam, dexmedetomidine sedation for pediatric MRI resulted in delayed sedation onset [Weighted mean differences $(\mathrm{WMD})=8.13 \mathrm{~min}, 95 \% \mathrm{CI}=4.64-11.63)$ and recovery times $(\mathrm{WMD}=5.22 \mathrm{~min}, 95 \% \mathrm{CI}=0.35-10.09)$ and decreased heart rate $(\mathrm{WMD}=-17.34$ beats per minute, $95 \% \mathrm{CI}=-22.42$ to -12.26 ). Of note, dexmedetomidine sedation was associated with a lower incidence of desaturation events $(\mathrm{RR}=0.42,95 \% \mathrm{CI}=0.20-0.86)$. There was no difference in sedation quality or incidence of sedation failure. They concluded that dexmedetomidine has the same sedation quality as other drugs but fewer respiratory events.

Kazuyoshi Aoyama

kazu.aoyama@utoronto.ca

1 Department of Critical Care Medicine, The Hospital for Sick Children, University of Toronto, Toronto, Canada

2 Department of Critical Care Medicine, Okayama University Hospital, Okayama, Japan

3 Department of Anesthesia and Pain Medicine, The Hospital for Sick Children, University of Toronto, Toronto, Canada

4 Department of Critical Care and Anesthesia, National Center for Child Health and Development, Tokyo, Japan

5 Program in Child Health Evaluative Sciences, The Hospital for Sick Children Research Institute, Toronto, Canada
Meanwhile, delayed sedation onset and recovery times are limitations of dexmedetomidine use for pediatric MRI sedation, but may represent a cautious administration regimen, consistent with the manufacturer's product insert. Since the use of dexmedetomidine for pediatric sedation was officially approved only in pediatric intensive care unit settings thus far [2], there is a multi-country ongoing trial of dexmedetomidine sedation for pediatric MRI in Japan, Canada, and the United States [3].

Procedural sedation is increasingly common in pediatrics [4]. The potential impact of sedative drugs on the pediatric population is important to understand due to developmental concerns [5]. The selection of proper drugs is essential when providing pediatric sedation and ideal features are: rapid predictable onset, short context-sensitive half-time, dose-dependent effect, rapid offset, maintenance of airway tone, spontaneous ventilation and hemodynamic status, no injection pain, and the existence of a reversal. Historically, midazolam and chloral hydrate were popular sedative drugs for pediatric sedation, though recently there is an increasing trend in the use of propofol and dexmedetomidine [4]. There are two existing meta-analyses which compared dexmedetomidine and propofol for pediatric MRI sedation [6, 7]. One study concluded that propofol should be encouraged for pediatric MRI given its better sedative effects and low incidence of emergence delirium [6] while the other study confirmed that dexmedetomidine had a prolonged recovery time [7]. There has been no sedative drug that has all the ideal features for pediatric MRI sedation until recently.

Remimazolam is a brand-new general anesthetic, which is an ultra-short-acting benzodiazepine metabolized by tissue esterases to an inactive compound. Remimazolam has a small steady-state volume of distribution with a short elimination half-life. Researchers strategically designed this novel drug to be metabolized by esterases to provide a preferable sedative profiles [8]. In the United States, European Union, 


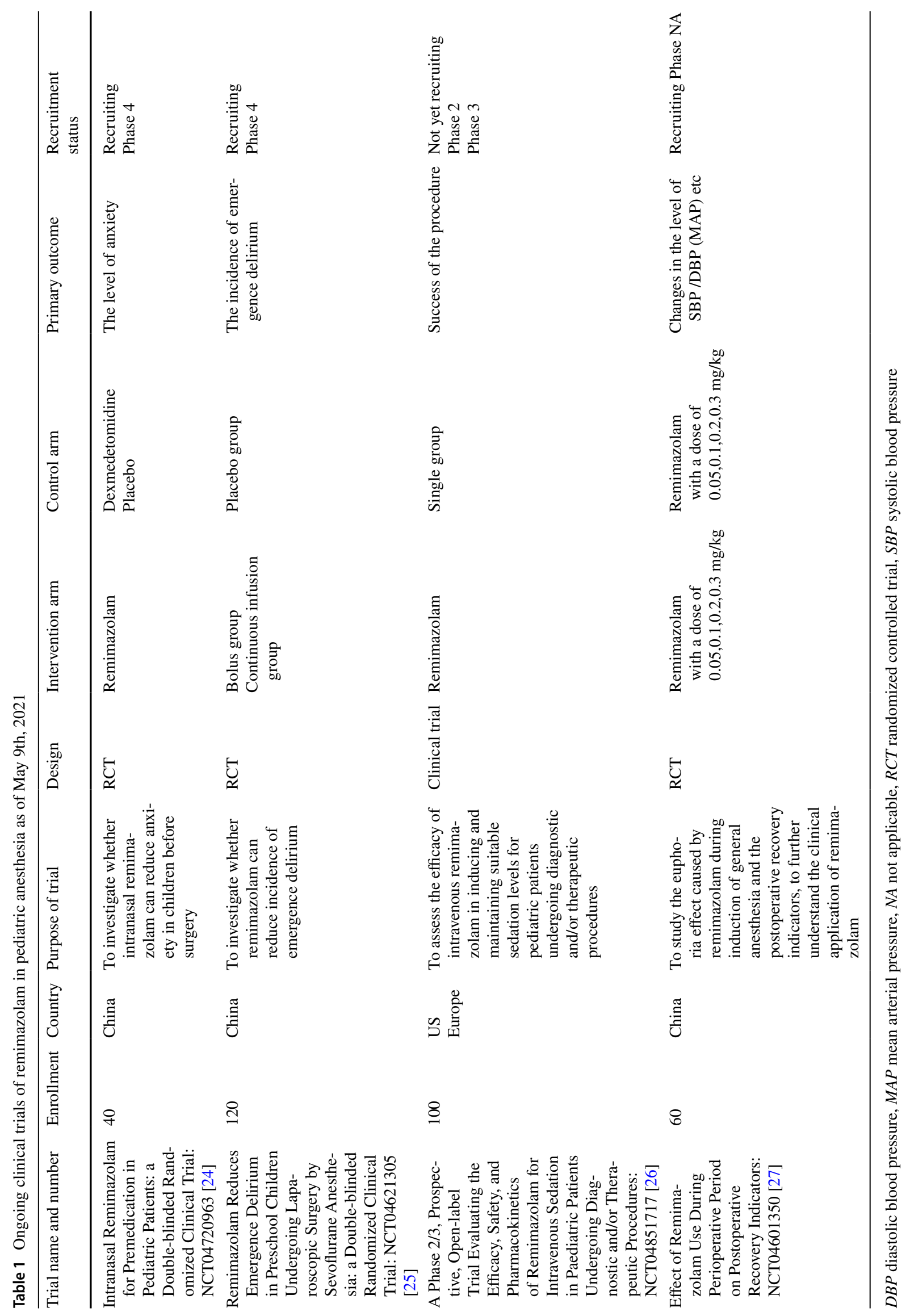


and China, remimazolam was initially approved for procedural sedation in adults [9-11]. The typical adult dose of remimazolam for procedural sedation is $5 \mathrm{mg}$ via intravenous injection over $1 \mathrm{~min}$ and then $2.5 \mathrm{mg}$ via intravenous injection over $15 \mathrm{~s}$ as a rescue dose [12, 13]. In Japan and South Korea, remimazolam was approved for general anesthesia in adults [11, 14-16]. The dose of remimazolam recommended for anesthesia induction is at an infusion rate of $12 \mathrm{mg} / \mathrm{kg} / \mathrm{hour}$, which is adjustable based on the patient's age and clinical background, and that for the maintenance phase is $1 \mathrm{mg} / \mathrm{kg} / \mathrm{hour}$, which is also adjustable to a maximum rate of $2 \mathrm{mg} / \mathrm{kg} /$ hour depending on clinical parameters (e.g., heart rate and blood pressure) [17]. Monitoring the electroencephalographic (EEG) index is desirable due to possible interpersonal variability; however, there are no reliable data available for appropriate ranges of EEG index at this point.

Remimazolam does not produce injection site pain which is common in propofol use (18.7\% of cases) [17]. The incidence of intraoperative hypotension events is lower with remimazolam (22\%) compared with propofol (49.3\%) [17]. There is no need for unplanned mechanical ventilation during procedural sedation with using remimazolam [18]. With respect to recovery, the mean time from the end of the procedure to full alertness using remimazolam is shorter [6.0 $\mathrm{min}$ (95\% CI 5.2-7.1)] compared to midazolam [12 $\mathrm{min}$ (95\%CI 5.0-15)] [13]. However, the mean time to extubation after discontinuing remimazolam infusion [19.2 min (Standard deviation; SD 10.8)] is longer than after discontinuing propofol [13.1 min (SD 6.5)] [17]. This finding may be a result of less clinical experience with remimazolam and may be overcome using flumazenil, which reverses remimazolam effects. Note there is no recommendation for the dose of flumazenil for remimazolam reversal [19] and it remains unknown whether reversal of remimazolam by flumazenil may or may not cause re-sedation later [19, 20]. The use of flumazenil is not inconsequential or without side effects, so routine reversal of benzodiazepine effects cannot be recommended at this stage. Therefore, future trials are warranted to investigate the efficacy and safety of flumazenil use as a reversal of remimazolam in pediatric sedation.

All evidence cited to this point is from studies in adults. We conducted a comprehensive literature search in MEDLINE, EMASE, and International Pharmaceutical Abstracts (April 21, 2021), containing sets of terms reflecting the medication (remimazolam) without language restriction, which yielded 150 abstracts (Supplementary online resource 1). Of the 150 abstracts, none was in the pediatric population. We further searched ongoing pediatric trials of remimazolam at ClinicalTrials.gov (May 9, 2021) and found 4 ongoing clinical trials of remimazolam in pediatric anesthesia [21], including sedation (summary in Table 1). Three trials are in
China, and one in the U.S. and Europe and is the only trial of pediatric sedation for diagnostic/therapeutic procedures.

Limitations of propofol sedation include injection site pain and respiratory and hemodynamic depression without a reversal agent. Conversely, remimazolam has a reversal agent and no injection site pain, so could be considered as the primary sedative medication for pediatric MRI in the future. Dexmedetomidine is also an option for pediatric MRI, especially for children with obstructive sleep apnea undergoing cine MRI and children requiring magnetoencephalography $[22,23]$. Future trials are warranted to investigate the safety and efficacy of remimazolam compared to propofol for pediatric MRI, and to also understand essential perioperative outcomes in children such as emergence delirium, postoperative nausea and vomiting, and respiratory events.

Supplementary Information The online version contains supplementary material available at https://doi.org/10.1007/s00540-021-02957-1.

Acknowledgements All authors acknowledge Ms. Marina Englesakis, MLIS at Library and Information Services, University Health Network for her support of literature search. KA acknowledges the Department of Anesthesiology and Pain Medicine, University of Toronto, and the Hospital for Sick Children, for secured academic time to conduct the current work as a recipient of Merit Awards.

Author contributions NS and KA conceived this paper. NS and KA wrote the initial and subsequent draft of the manuscript, which TE and YS commented and edited. All authors approved the final version.

Funding This work was supported by Perioperative Services Facilitator Grant Program 2020-2021, and Outcomes Research Award 2020 2021, Department of Anesthesia and Pain Medicine, Hospital for Sick Children (KA).

\section{Declarations}

Conflict of interest TE and YS are engaged in an industry trial by Pfizer described in the main text [3], which did not affect this publication. Hence, all authors declare that they have no competing interests related to this publication.

\section{References}

1. Kim JY, Kim KN, Kim DW, Lim HJ, Lee BS. Effects of dexmedetomidine sedation for magnetic resonance imaging in children: a systematic review and meta-analysis. J Anesth. 2021. https://doi. org/10.1007/s00540-021-02946-4.

2. Takeuchi M, Nemoto S, Suzuki Y, Takahashi N, Takenaka N, Takata A, Kobayashi M. Age-specific dose regimens of dexmedetomidine for pediatric patients in intensive care following elective surgery. Pediatr Crit Care Med. 2021. https://doi.org/10.1097/ PCC.0000000000002730.

3. Safety and Efficacy of Dexmedetomidine (DEX) for sedation of subjects $\geq 1$ Month to $<17$ Years Undergoing MRI scans - full text View - ClinicalTrials.gov [Internet]. https://clinicaltrials.gov/ct2/ 
show/NCT04237792?term $=$ dexmedetomidine $\% 2 \mathrm{C}+$ MRI \&draw $=$ $2 \&$ rank $=2$. (Accessed date 18 May 2018)

4. Kamat PP, McCracken CE, Simon HK, Stormorken A, Mallory M, Chumpitazi CE, Cravero JP. Trends in outpatient procedural sedation: 2007-2018. Pediatrics. 2020;145.

5. Toney M, Pattishall S, Garber M. The time is now: standardized sedation training for pediatric hospitalists. Pediatrics. 2020. https://doi.org/10.1542/peds.2020-0446.

6. Zhou Q, Shen L, Zhang X, Li J, Tang Y. Dexmedetomidine versus propofol on the sedation of pediatric patients during magnetic resonance imaging (MRI) scanning: a meta-analysis of current studies. Oncotarget. 2017. https://doi.org/10.18632/oncotarget. 22271.

7. Fang HW, Yang L, Wang XR, Zhu H. Clinical efficacy of dexmedetomidine versus propofol in children undergoing magnetic resonance imaging: a meta-analysis. Int J Clin Exp Med. 2015;8:11881-9.

8. Kilpatrick GJ, McIntyre MS, Cox RF, Stafford JA, Pacofsky GJ, Lovell GG, Wiard RP, Feldman PL, Collins H, Waszczak BL, Tilbrook GS. CNS 7056: a novel ultra-short-acting Benzodiazepine. Anesthesiology. 2007;107:60-6.

9. Mahmoud M, Mason KP. A forecast of relevant pediatric sedation trends. Curr Opin Anaesthesiol. 2016;29:56-67.

10. Worthington MT, Antonik LJ, Goldwater R, Lees JP, WilhelmOgunbiyi K, Borkett KM, Mitchell MC. Phase 1B dose-finding study of multiple doses of remimazolam (CNS 7056) in volunteers undergoing colonoscopy. Anesth Analg. 2013;117:1093-100.

11. Paion - About Remimazolam [Internet]. https://www.paion.com/ remimazolam/indikationen/leitsubstanz-remimazolam/. (Accessed date 9 May 2021)

12. Remimazolam uses, side effects and warnings - Drugs.com. https://www.drugs.com/mtm/remimazolam.html. (Accessed date 13 May 2021)

13. Pastis NJ, Yarmus LB, Schippers F, Ostroff R, Investigators $P$, Chen A, Akulian J, Wahidi M, Shojaee S, Tanner NT, Callahan SP, Feldman G, Lorch DG Jr, Ndukwu I, Pritchett MA, Silvestri GA, PAION Investigators. Safety and efficacy of remimazolam compared with placebo and midazolam for moderate sedation during bronchoscopy. Chest. 2019;155:137-46.

14. Doi M, Hirata N, Suzuki T, Morisaki H, Morimatsu H, Sakamoto A. Safety and efficacy of remimazolam in induction and maintenance of general anesthesia in high-risk surgical patients (ASA Class III): results of a multicenter, randomized, double-blind, parallel-group comparative trial. J Anesth. 2020;34:491-501.

15. Masui K. Remimazolam besilate, a benzodiazepine, has been approved for general anesthesia!! J Anesth. 2020;34:479-82.

16. Hirata N, Hayamizu K, Yamakage M. How to administer remimazolam for anesthesia induction. J Anesth. 2020;34:962.
17. Doi M, Morita K, Takeda J, Sakamoto A, Yamakage M, Suzuki T. Efficacy and safety of remimazolam versus propofol for general anesthesia: a multicenter, single-blind, randomized, parallelgroup, phase IIb/III trial. J Anesth. 2020;34:543-53.

18. Pambianco DJ, Borkett KM, Riff DS, Winkle PJ, Schwartz HI, Melson TI, Wilhelm-Ogunbiyi K. A phase IIb study comparing the safety and efficacy of remimazolam and midazolam in patients undergoing colonoscopy. Gastrointest Endosc. 2016;83:984-92.

19. Godai K. What are mechanisms of re-sedation caused by remimazolam? J Anesth. 2021;35:466.

20. Yamamoto T, Kurabe M, Kamiya Y. Re-sleeping after reversal of remimazolam by flumazenil. J Anesth. 2021;35:322.

21. Search of: remimazolam I Child - List Results - ClinicalTrials. gov [Internet]. https://clinicaltrials.gov/ct2/results?cond=remim azolam\&age_v $=\&$ age $=0 \&$ gndr $=\&$ type $=\& r s l t=\&$ Search $=$ Apply. (Accessed date 9 May 2021)

22. Mahmoud M, Gunter J, Donnelly LF, Wang Y, Nick TG, Sadhasivam S. A comparison of dexmedetomidine with propofol for magnetic resonance imaging sleep studies in children. Anesth Analg. 2009;109:745-53.

23. Konig MW, Mahmoud MA, Fujiwara H, Hemasilpin N, Lee KH, Rose DF. Influence of anesthetic management on quality of magnetoencephalography scan data in pediatric patients. Paediatr Anaesth 2009; 19:507-12.

24. Intranasal remimazolam for premedication in pediatric patient - full text view - ClinicalTrials.gov [Internet]. https://clinicaltr ials.gov/ct $2 /$ show $/$ NCT04720963? cond $=$ remimazolam $\&$ age $=0 \&$ draw $=2 \&$ rank $=1$. (Accessed date 13 May 2021)

25. Remimazolam reduces emergence delirium in preschool children undergoing laparoscopic surgery by sevoflurane anesthesia - full text view - ClinicalTrials.gov [Internet]. https://clinicaltrials.gov/ ct $2 /$ show $/$ NCT04621305 cond $=$ remimazolam\&age $=0 \& d r a w=2 \&$ rank=2. (Accessed date 13 May 2021)

26. Investigation of remimazolam in children undergoing sedation for medical procedures - full text view - ClinicalTrials.gov [Internet]. https://clinicaltrials.gov/ct2/show/NCT04851717? cond=remim azolam $\&$ age $=0 \&$ draw $=2 \&$ rank $=3$. (Accessed date 13 May 2021)

27. Effect of remimazolam use during perioperative period on postoperative recovery indicators - full text view - ClinicalTrials.gov [Internet]. https://clinicaltrials.gov/ct2/show/NCT04601350? cond $=$ remimazolam $\&$ age $=0 \&$ draw $=2 \&$ rank $=4$. (Accessed date 13 May 2021)

Publisher's Note Springer Nature remains neutral with regard to jurisdictional claims in published maps and institutional affiliations. 